\title{
ANTIBIORÉSISTANCE A L'ÉCHELLE « UNE SEULE SANTÉ » : STRATÉGIES, ENJEUX ET OPPORTUNITÉS
}

\author{
ANTIMICROBIAL RESISTANCE AND ONE HEALTH: STRATEGIES, \\ CHALLENGES AND OPPORTUNITIES
}

par Yohann LACOTTE', Marie-Cécile PLOY'

(Communication effectuée le 7 octobre 2021, manuscrit accepté le 27 juillet 2021)

\begin{abstract}
RÉSUMÉ
La Covid-19 nous a rappelé les dangers liés aux zoonoses et nous invite à repenser notre vision de la Santé. Dans un monde où hommes, animaux et écosystèmes sont liés, seule une approche collective basée sur le partage des savoirs et expertises, dite Une Seule Santé ou One Health, peut espérer endiguer les menaces sanitaires à venir. Parmi ces menaces, l'antibiorésistance serait responsable, chaque année, de plus de 30000 décès en Europe. Ces 20 dernières années, l'antibiorésistance a su fédérer des acteurs de la santé humaine, animale et des sciences de l'environnement à différentes échelles et selon diverses stratégies. Néanmoins, malgré des efforts évidents, le One Health se confronte encore à plusieurs enjeux pour s'ancrer dans les pratiques. Pour s'institutionnaliser, il doit apprendre à se structurer et à fédérer ses acteurs autour d'enjeux communs tels que l'échange de bonnes pratiques ou de données. Il doit également apprendre à mieux incorporer la dimension environnementale dans son action. Il doit enfin capitaliser sur l'éducation et la sensibilisation pour créer une dynamique One Health durable.
\end{abstract}

Mots-Clés : Une Seule Santé, One Health, santé publique, santé animale, environnement, résistance aux antibiotiques.

\begin{abstract}
The Covid-19 pandemic reminds us the danger related to zoonosis and invites us to think on our vision of Health. In a world where humans, animals and ecosystems are all connected, several health threats can only be addressed through a multidisciplinary One Health approach based on knowledge and skill sharing. Among these threats, antimicrobial resistance silently kills 30000 people each year in Europe. During the past twenty years, antimicrobial resistance managed to gather people from the human, animal and environmental field at different scales and following different strategies. However, despite evident efforts, the One Health concept still faces challenges to anchor itself successfully in daily practices. Structuration efforts are needed to allow practice and data sharing among professionals from different sectors. The environmental dimension is also often neglected especially in the surveillance field. Finally, education and awareness raising need to be improved to create a sustainable One Health dynamic.

Keywords: One Health, public health, animal health, environment, antimicrobial resistance.
\end{abstract}

\section{L'IMPORTANCE DU « ONE HEALTH » À LA LUMIÈRE DU COVID-19}

L'épidémie de Covid-19 nous a rappelé les dangers liés aux maladies transmissibles. Plus que jamais ces maladies représentent une menace globale alors même que les agents pathogènes qui les causent peuvent se déplacer d'un continent à l'autre dans des délais inférieurs à leur période d'incubation. Bactéries, virus, parasites et champignons ne connaissent aucune frontière. La crise liée à la diffusion mondiale du virus SARS-CoV-2, responsable de la Covid-19, en est l'illustration parfaite. En quelques mois, une maladie infectieuse émergente a réussi à se propager dans le monde entier, contaminant plus de 150 millions de personnes et causant plus de 3 millions de décès (Wu et al. 2021). Avant le SARS-CoV2, plusieurs maladies infectieuses avaient déjà menacé la planète comme la peste, la tuberculose, la rage, et plus récemment, la grippe aviaire, Ebola, Zika, Chikungunya... De façon intéressante, toutes ces infections partagent une caractéristique commune : elles font

1 Université Limoges, INSERM, CHU Limoges, UMR 1092, 2, rue du Docteur Marcland, 87025 Limoges. Courriel : yohann.lacotte@inserm.fr, marie-cecile.ploy@unilim.fr 
partie de la grande famille des zoonoses. Toutes ont pour origine, un hôte animal. C'est d'ailleurs le cas de $60 \%$ des maladies infectieuses connues et de $75 \%$ des nouvelles maladies émergentes (Jones et al. 2008 ; Woolhouse \& Gowtage-Sequeria, 2005). Chaque année, les zoonoses seraient responsables de 2,5 milliards d'infections et de 2,7 millions de décès dans le monde (Salyer et al. 2017). L'incidence de ces maladies n'a cessé de croitre au cours des dernières décennies du fait de l'activité humaine. La pression de l'espèce humaine sur son environnement (extension des terres agricoles, déforestation) accroit le risque de contact entre l'homme et les animaux. A plus long terme, le réchauffement climatique pourrait également modifier les aires de présence de certaines espèces sauvages dont les moustiques vecteurs de nombreuses maladies parasitaires (Booth, 2018). L'importance du réservoir animal dans l'émergence des maladies infectieuses nous invite à repenser notre vision de la Santé et à reconnaitre l'interdépendance entre hommes et animaux. De ce constat est né un premier concept, le "One Medicine ", proposé par les médecins prussien et canadien, R. Virchow (1821-1902) et W. Osler (1849-1919). Plus tard, ce concept évoluera pour intégrer la santé des écosystèmes environnementaux et sera rebaptisé "One World, One Health " (Un Monde, Une Seule Santé), plus simplement abrégé Une Seule Santé ou "One Health ». Ce concept prône une approche collective et multidisciplinaire entre professionnels issus des trois secteurs humain, animal et environnement - pour prévenir et contrôler plus efficacement les menaces sanitaires émergentes. Le "One Health" capitalise sur la mise en commun des savoirs et expertises pour mieux appréhender les interactions complexes entre ces trois réservoirs. La notion de One Health a été formellement introduite par la Wildlife Conservation Society en 2004, lors d'un symposium intitulé "One World, One Health ». Lors de cette réunion, la Société publie une série de 12 principes, les " principes de Manhattan " qui recommandent l'utilisation d'une approche holistique et multidisciplinaire pour gérer au mieux les maladies épidémiques et zoonotiques ainsi que préserver les écosystèmes au bénéfice de la santé humaine et animale (Wildlife Conservation Society, 2004). Ces principes fondateurs ont conduit, en 2010, à une alliance stratégique entre l'Organisation mondiale de la santé (OMS), l'Organisation pour l'alimentation et l'agriculture (FAO) et l'Organisation mondiale de la santé animale (OIE). Cette association vise à proposer une démarche coordonnée et un partage de responsabilités dans la lutte contre les menaces épidémiques et zoonotiques. Fort du soutien de la Banque mondiale, cette alliance tripartite a initié de nombreuses approches collaboratives One Health pour détecter et prévenir précocement les risques sanitaires. En 2018, le Programme des Nations unies pour l'environnement (UNEP) rejoint l'alliance tripartite pour former le " tripartite plus " et mieux couvrir la dimension environnementale du One Health. Parmi les initiatives récentes de ce "tripartite plus », on peut notamment citer la création d'un comité international d'experts One Health, le OHHLEP (One Health High-Level Expert Panel) chargé de coordonner la réponse One Heath à l'échelle du globe. La France, quant à elle, se positionnait dès 2011 sur le concept One Health en proposant une vision intégrative de la santé (Ministère des affaires étrangères et européennes, 2011). L'Europe fait également figure de bon élève sur le plan international avec des initiatives structurantes permettant de faire des liens entre les professionnels des trois secteurs, notamment le "One Health European Joint Programme " (OHEJP), rassemblant 44 partenaires issus de 22 pays Européens (https://onehealthejp.eu/). Initialement centré sur la gestion du risque infectieux, le concept One Health s'est depuis élargi à d'autres enjeux sanitaires tels que les contaminants biologiques et chimiques de la chaine alimentaire (Jeggo Martyn et al. 2014) ou des enjeux climatiques et environnementaux (Zinsstag et al. 2018). Aujourd'hui, l'une des principales incarnations

du

One

Health reste la lutte contre l'antibiorésistance.

\section{ANTIBIORÉSISTANCE ET ONE HEALTH}

Depuis les années 70, la résistance aux antibiotiques s'est érigée comme l'un des enjeux majeurs de Santé publique en témoigne l'évolution du nombre de publications sur le sujet entre 1960 et 2020 (Figure 1)

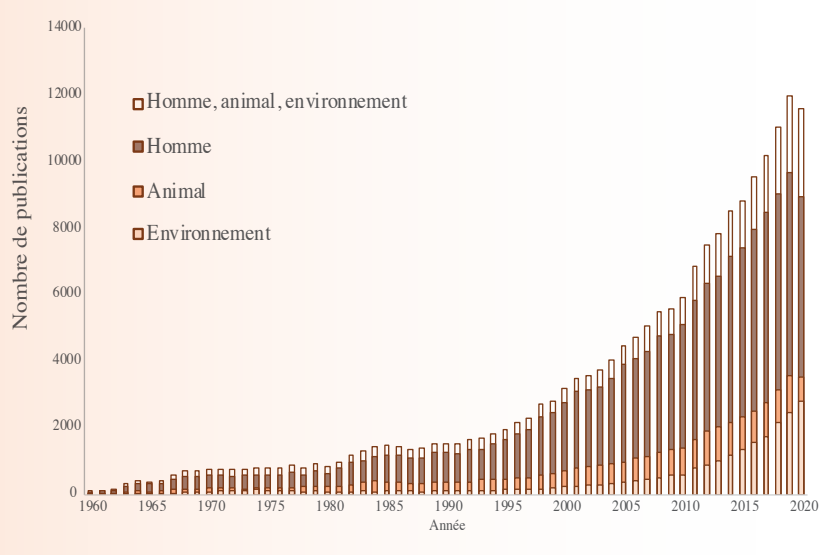

Figure 1 : Evolution du nombre de publications sur PubMed entre 1960 et 2020 sur la résistance aux antibiotiques chez l'homme, les animaux et dans l'environnement (https://pubmed.ncbi.nlm.nih.gov/)

Requêtes utilisées : homme $=$ (antibiotic) AND (resistance) AND (human), animal $=$ (antibiotic) AND (resistance) AND (animal), environnement $=($ antibiotic) AND (resistance) AND (environement), homme, animal ou environnement $=$ (antibiotic) AND (resistance) AND ((human) OR (animal) OR (environment).

Cette figure illustre bien la différence de temporalité en recherche sur l'antibiorésistance dans les 3 secteurs. C'est seulement à partir de 1990 que la recherche sur l'antibiorésistance en médecine animale s'est accélérée ; et seulement à partir des années 2000 pour la recherche sur l'antibiorésistance dans l'environnement.

Les antibiotiques font partie des clés de voûte de la médecine moderne. Ils sont indispensables pour traiter nombres d'infections et prévenir ces dernières lors de chirurgies, actes invasifs ou traitements immuno-modulateurs. Pourtant l'utilisation des antibiotiques est aujourd'hui menacée alors que de plus en plus de bactéries développent des résistances et que de moins en moins d'antibiotiques innovants sont mis sur le marché. En 2015, plus de 670000 infections et 33000 décès attribuables à des bactéries résistantes ont été recensés chez l'homme en Europe (Cassini et al. 2019). En 2018, une étude prédictive de l'OCDE (Organisation de coopération et de développement économiques) estimait qu'à l'horizon 2050, plus de 2,4 millions 
de personnes auront succombé des suites d'une infection par une bactérie résistante en Europe, en Australie et aux Etats-Unis (OECD, 2018). En 2050, la résistance aux différents antimicrobiens (antibiotiques, antiviraux, antifongiques, antiparasitaires) pourrait être la principale cause de mortalité à l'échelle du globe (O’Neill, 2016). Lutter efficacement contre l'antibiorésistance nécessite une approche One Heath globale et coordonnée. En effet, les gènes permettant de résister aux antibiotiques sont le plus souvent véhiculés par des éléments génétiques mobiles (plasmides, transposons, séquences d'insertions, cassettes d'intégrons, ...) capables de se déplacer d'une bactérie à une autre par transferts horizontaux. Si les résistances sont capables de se disséminer au sein des écosystèmes bactériens, les bactéries résistantes sont aussi elles-mêmes capables de se disséminer entre les trois réservoirs : humain, animal et environnement. Les transferts de résistances entre l'homme et l'animal sont bien documentés. Ils peuvent se faire dans les deux sens à la faveur de contacts rapprochés entre l'homme et l'animal (Garcia-Graells et al. 2012 ; Haenni et al. 2012 ; Köck et al. 2012 ; Poirel et al. 2012) et dans le sens animal-homme via la chaine alimentaire (Yaici et al. 2017). Par ailleurs, humains et animaux rejettent des bactéries dans l'environnement, via leur fèces notamment, faisant de ce dernier, un immense réservoir de gènes de résistance. Le risque sanitaire posé par celui-ci est néanmoins encore mal connu à l'heure actuelle. Quel que soit le réservoir considéré, la dissémination et la persistance des résistances sont d'autant plus facilitées par l'activité anthropique. L'utilisation abusive des antibiotiques en médecine humaine et animale ainsi que certaines pratiques agricoles et industrielles (rejets d'antibiotiques, biocides, métaux lourds, ...) génèrent des pressions de sélection capables de sélectionner ou co-sélectionner les résistances. La Figure 2 illustre bien la complexité des cycles de transmission entre les trois réservoirs et la multiplicité des facteurs qui favorisent l'antibiorésistance. Raisonnablement, seule une approche One Health pluridisciplinaire entre professionnels de la santé humaine, animale et environnementale peut espérer endiguer durablement ce phénomène (White \& Hughes, 2019). Fort heureusement la mobilisation autour de l'antibiorésistance a été à la hauteur de l'enjeu ces dernières années et les initiatives One Health se sont multipliées à différentes échelles.

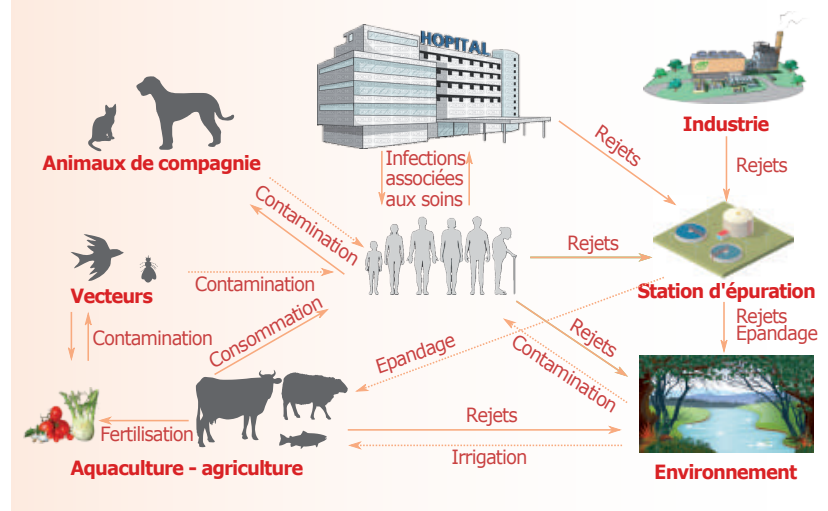

Figure 2 : Cycle de transmission des résistances entre hommes, animaux et environnement. Les flèches pleines représentent les cycles de transmission documentés. Les flèches pointillées représentent des cycles de transmissions encore mal caractérisés.

\section{LES STRATÉGIES ONE HEALTH POUR LUTTER CONTRE L'ANTIBIORÉSISTANCE}

\section{A l'échelle du globe}

C'est à la faveur du tripartite OMS, FAO, OIE que la réponse au fléau de l'antibiorésistance a commencé à se structurer à l'échelle du globe. En 2012, le tripartite se dote d'un secrétariat commun dédié à la lutte contre l'antibiorésistance. Ce secrétariat veut d'une part sensibiliser au problème de l'antibiorésistance et en faire un sujet politique, puis d'autre part, ambitionne de coordonner et structurer la réponse globale à cet enjeu majeur de santé publique. Cet engagement se traduit en 2016, par la publication du premier plan d'action mondial One Health de lutte contre l'antibiorésistance. Ce plan d'action repose sur l'engagement de l'ensemble de la société et des acteurs du One Health. Il prévoit la mise en place de mesures durables pour prévenir les infections et limiter l'émergence de résistance (WHO, 2016). Ce plan définit également 5 enjeux stratégiques pour lutter effacement contre l'antibiorésistance : (i) le renforcement des connaissances par la surveillance et la recherche, (ii) la diminution de l'incidence des infections via des mesures d'hygiène et de prévention, (iii) l'amélioration des pratiques d'utilisation des antibiotiques en santé humaine et animale, (iv) le renforcement des formations, outils éducatifs et de la communication autour de l'antibiorésistance et enfin (v) un soutien économique durable. Par la suite, le tripartite se dote d'outils pour mettre en ouvre ce plan d'action : des guides de bonnes pratiques aussi bien en santé humaine qu'animale, des outils de formations (cours en ligne type MOOC notamment) et des outils de surveillance locaux, nationaux ou internationaux. Parmi les outils de surveillance qui incarnent au mieux l'approche One Health, on peut notamment citer le récent protocole "tricycle " de l'OMS qui prévoit une surveillance simple, multi sectorielle et intégrative des Escherichia coli producteurs de bêta-lactame à spectre étendu (BLSE) chez l'homme (données communautaires et hospitalières dans capitales ou grandes villes), chez les animaux (volailles destinées à la consommation dans les mêmes villes) et dans l'environnement (échantillons de rivière en amont et aval des villes sélectionnées, les effluents d'abattoirs et des hôpitaux dans celles-ci). Par ailleurs, en décembre 2016, les chefs d'État du monde entier se sont engagés à mettre en place des plans d'action nationaux multisectoriels "One Health " dans une résolution commune des Nations-Unies. Depuis l'antibiorésistance a souvent été discutée dans le cadre du G20 ou du G7. Elle a notamment été l'un des sujets phares du dernier sommet du G7 qui s'est tenu en Grande-Bretagne en Juin 2021 (G7, 2021).

\section{À l'échelle Européenne}

L'Europe a très tôt pris la mesure du problème de l'antibiorésistance, en témoigne la stratégie communautaire de 2001 de lutte contre la résistance antimicrobienne (Commission Européenne, 2001). Cette politique européenne se renforce en novembre 2011 via l'engagement de la Commission Européenne à développer un plan d'action européen «One 
Health " de lutte contre l'antibiorésistance. Ce plan d'action, publié en 2017, prévoit de faire de l'Europe une région d'excellence tant sur le domaine des pratiques que de la recherche et repose sur la collaboration et les échanges entre les États Membres et les acteurs du One Health (European Commission, 2017). En outre, l'Union européenne invite ses États Membres à mettre en place des plans d'action nationaux One Health à l'horizon mi-2017, dans le cadre des Conclusions du Conseil sur les prochaines étapes de la lutte contre la résistance aux antimicrobiens de juin 2016 (Commission Européenne, 2016). Pour aider les États Membres à améliorer leurs pratiques, la Commission Européenne et l'agence exécutive pour les consommateurs, la santé, l'agriculture et l'alimentation (CHAFEA) lancent en 2017 une action conjointe européenne pour lutter contre la résistance aux antibiotiques et les infections associées aux soins : l'EU-JAMRAI (https://eu-jamrai.eu/). Cette action conjointe, coordonnée par la France, rassemble 44 partenaires européens, issus de 26 pays, et plus de 40 parties prenantes impliquées dans la lutte contre l'antibiorésistance et les infections associées aux soins. Cette action conjointe ambitionne de renforcer les synergies entre les États Membres et à mettre en place des politiques de santé publique One Health durables en Europe. L'EU-JAMRAI a notamment développé le premier symbole représentant la lutte contre l'antibiorésistance, qui, à l'instar du ruban rouge pour le VIH, devrait permettre de sensibiliser le grand public au problème de l'antibiorésistance. Dans une démarche One Health, l'EU-JAMRAI a notamment travaillé à la structuration d'un réseau de surveillance vétérinaire européen, baptisé EARS-Vet. EARS-Vet propose une approche harmonisée et coordonnée pour surveiller la résistance aux antibiotiques chez les animaux malades en Europe en s'appuyant sur les systèmes nationaux déjà en place (Madec et al. 2021a, 2021b). EARS-Vet viendrait ainsi complémenter habilement le réseau européen de surveillance de la résistance aux antimicrobiens chez l'homme (EARS-Net) porté par le centre européen de prévention et de contrôle des maladies (ECDC, https://www.ecdc.europa.eu/en/about-us/partnershi ps-and-networks/disease-and-laboratory-networks/ears-net). Sur la base d'un travail de structuration et de méthodologie réalisé dans le cadre de l'EU-JAMRAI, EARS-Vet espère pouvoir lancer prochainement une étude pilote en Europe. A plus long terme, ce système de surveillance pourrait être repris par des agences de l'UE (Autorité européenne de sécurité des aliments, EFSA, par exemple), si elles recevaient le mandat de coordonner la surveillance des résistances dans les agents pathogènes animaux (par exemple sous l'égide de la loi sur la santé animale). Cela garantirait l'intégration du système EARS-Vet dans le paysage européen et permettrait le lancement d'initiatives connexes contribuant à renforcer la surveillance One Health de la résistance en Europe. A l'initiative de l'ECDC, l'antibiorésistance dispose également depuis 2008 d'une journée européenne de sensibilisation, le 18 novembre de chaque année. Cet événement, souvent étendu à toute la semaine du 18 novembre, est l'occasion pour les nombreuses parties prenantes impliquées dans la lutte contre l'antibiorésistance de communiquer autour de leurs activités et de sensibiliser le grand public à cet enjeu majeur de santé publique.

\section{En France}

En France, la lutte contre l'antibiorésistance en médecine humaine a débuté en 2001 avec le premier plan national pour préserver l'efficacité des antibiotiques (2001-2005), suivi d'un second (2007-2010) puis en 2011 d'un plan national d'alerte sur les antibiotiques (Ministère chargé de la Santé, 2011). Ces plans d'action, bien que ne mentionnant pas encore l'approche One Health, incorporaient déjà quelques mesures transdisciplinaires, notamment des mesures de bonne pratique d'utilisation en santé humaine et animale pour réduire les pressions de sélection antibiotiques. C'est en 2016 que la France publie son premier plan d'action véritablement One Health par le biais d'une feuille de route interministérielle visant à maitriser l'antibiorésistance (Comité interministériel pour la Santé, 2016). Cette feuille de route s'appuie sur les recommandations du rapport Carlet et s'articule autour de 13 mesures phares. Ces différents plans ont notamment conduit, en 2012, à la mise en place par le ministère de l'Agriculture et de l'Alimentation du premier plan "Ecoantibio ». Ce plan sur 5 ans avait pour objectif de réduire la consommation d'antibiotiques à usage vétérinaire de $25 \%$ et à limiter le recours à des antibiotiques jugés critiques en médecine humaine. Il reposait sur l'utilisation de traitements et mesures alternatives aux antibiotiques ainsi que sur des mesures réglementaires et incitatives pour limiter les mauvaises pratiques. La collecte des données de consommation et de résistance est assurée par le réseau RESAPATH, sous l'égide de l'agence nationale de sécurité sanitaire de l'alimentation, de l'environnement et du travail (ANSES). Ce plan marque l'engagement fort de la profession vétérinaire dans la lutte contre l'antibiorésistance. Il se traduisait en 2017 par une diminution de plus $37 \%$ de la consommation globale d'antibiotiques en médecine vétérinaire et une réduction drastique du recours aux céphalosporines de $3^{\text {ème }}$ et $4^{\text {ème }}$ génération $(-81 \%)$ et fluoroquinolones (-75\%) (Moulin et al. 2017). Depuis 2016, l'utilisation à usage préventif de ces antibiotiques, jugés d'importance critique pour la médecine humaine par l'OMS (WHO, 2019), est proscrite en France. La diminution de la consommation d'antibiotiques critiques en médecine animale s'accompagne, depuis 2013, d'une diminution de la résistance à ces mêmes antibiotiques. En 2019, les proportions d'isolats de E. coli résistants aux céphalosporines de $3^{\text {ème }}$ et $4^{\text {ème }}$ génération (C3G/C4G) et aux fluoroquinolones étaient respectivement inférieures à $5 \%$ et $8 \%$ toute espèce considérée (ANSES, 2020). En 2017, "Ecoantibio2 " est lancé pour poursuivre les efforts engagés à l'horizon 2021, consolider les acquis et évaluer l'impact du précédent plan Ecoantibio. En santé humaine, le premier plan national pour préserver l'efficacité des antibiotiques et sa célèbre campagne publicitaire « Les antibiotiques, c'est pas automatique " ont permis de réduire de 10\% la consommation d'antibiotiques en ville et de $22 \%$ la consommation d'antibiotiques dans les hôpitaux entre 2000 et 2013 (ANSM, 2014). Depuis la consommation est relativement stable autour de 23 doses pour 1000 habitants et par jour en ville et 1,7 doses pour 1000 habitants et par jour dans les établissements de santé (Santé Publique France, 2019). D’un point de vue plus qualitatif, la consommation de $\mathrm{C} 3 \mathrm{G} / \mathrm{C} 4 \mathrm{G}$ et de fluoroquinolones a largement diminué en ville entre 2008 et 2018 (-37\% et -36\%, 
respectivement). En établissements de santé, si la consommation de fluoroquinolones a diminué de $24 \%$ sur cette même période, la consommation de $\mathrm{C} 3 \mathrm{G} / \mathrm{C} 4 \mathrm{G}$ a elle augmenté de $40 \%$. La diminution globale de la consommation en C3G/C4G et fluoroquinolones s'est faite au profit d'antibiotiques générant moins de résistance et recommandés par les règles de bon usage notamment l'amoxicilline $(+55 \%$ de consommation en ville et + 6\% en établissement de santé) (Santé Publique France, 2019). En médecine humaine, l'évolution des résistances est plus contrastée. Dans la communauté, la résistance aux fluoroquinolones chez E. coli est en baisse depuis 2013 et celle aux C3G/C4G en baisse depuis 2015. Dans les établissements de santé, la résistance aux C3G/C4G a augmenté de 2008 à 2017 (2\% de E. coli résistants en 2008 à $11 \%$ en 2017) et diminue légèrement depuis. La résistance aux fluoroquinolones chez $E$. coli est, elle, restée globalement stable depuis 2008 (environ 15\% d'isolats résistants). Depuis 2018, l'antibiorésistance fait également l'objet d'un programme prioritaire de recherche en France, porté par l'Inserm (https://ppr-antibioresistance.i nserm. $\mathrm{fr} / \mathrm{fr} /$ ). Ce programme de recherche, financé à hauteur de 40 millions par le troisième programme d'investissements d'avenir, a pour objectif de favoriser l'interdisciplinarité et de faire émerger une synergie des recherches fondamentales, environnementales, cliniques, de santé publique, et vétérinaires dans un esprit véritablement One Health. Véritable incarnation de l'esprit One Health, la France dispose également d'une cellule de veille hepta-académique sur l'antibiorésistance. Cette cellule rassemble des membres des académies de l'Agriculture, de Chirurgie, de Chirurgie Dentaire, de Médecine, de Pharmacie, Vétérinaire ainsi que des Sciences et illustre bien le caractère transdisciplinaire nécessaire pour lutter contre l'antibiorésistance.

\section{ENJEUX ET OPPORTUNITÉS POUR AMÉLIO- RER LES PRATIQUES DE ONE HEALTH.}

Les exemples détaillés ci-dessus illustrent la dynamique One Health créée autour de l'antibiorésistance, sujet qui fait aujourd'hui office d'exemple et reste bien en avance, en terme d'engament One Health, par rapport aux zoonoses ou infections émergentes. Néanmoins, malgré des réussites évidentes dans le domaine de l'antibiorésistance, le One Health peine encore à s'ancrer durablement dans les pratiques.

\section{Améliorer la communication et les échanges entre les trois secteurs grâce à des efforts de structuration et aux sciences sociales}

A l'heure actuelle, l'un des principaux freins à la mise en œuvre du concept One Health reste sans aucun doute le manque de communication entre les différents acteurs impliqués. Un récent questionnaire sur les pratiques One Health en Europe montre que plus de $18 \%$ des répondants (171 participants issus de 34 pays représentant les 3 secteurs) considèrent le travail en silo comme la principale limite au One Health, devant le manque de ressources ( $16 \%$ des répondants), les problèmes institutionnels (11\%) et le manque de formation (8\%) (Chiesa et al. 2021). Ce risque de silotage est systémique du One Health.
Historiquement, santé humaine, animale et l'environnement ont toujours été dissociés. Les professionnels des trois secteurs répondent donc à des besoins et enjeux différents. Rassembler des acteurs si différents autour d'enjeux communs reste difficile sans des efforts majeurs de structuration.

Cette structuration passe par la création de réseaux locaux, nationaux ou internationaux réunissant des professionnels issus des 3 secteurs autour d'enjeux communs transdisciplinaires tels que la surveillance, l'analyse et la gestion du risque infectieux ou encore la prévention. Cette structuration repose aussi sur le rapprochement des différents ministères ou agences en charge des acteurs du One Health pour limiter certaines barrières institutionnelles. Elle passe, enfin, par la création de réseaux de recherche transdisciplinaire nationaux et internationaux regroupant des experts des 3 secteurs permettant de mieux caractériser les interactions entre écosystèmes et les facteurs de risque infectieux. Cette structuration doit aussi se faire en lien avec les sciences sociales. Pour améliorer la communication entre les acteurs des différents secteurs, il est notamment crucial d'identifier les barrières et facilitateurs aux efforts de structuration. Dans un cadre où les acteurs du One Health n'utilisent pas nécessairement le même vocabulaire, le recours aux sciences du langage peut également être pertinent pour renforcer les initiatives de structuration. Bien consciente de ces enjeux, la France a initié en 2020 dans le cadre du programme prioritaire de recherche, des appels à projets structurants, coordonné par l'Inserm, qui devraient permettre de créer (i) un méta-réseau de professionnels "One Health ", (ii) un observatoire des discours, des normes et pratiques liés à l'antibiorésistance en France et (iii) une plateforme de données microbiologiques et multi-omiques - homme, animal et environnement - intégrées et interopérables ; trois éléments qui devraient aider à favoriser les synergies entre secteurs.

\section{Favoriser les échanges de données entre secteurs pour une meilleure analyse du risque infectieux}

L'un des principaux enjeux autour de la structuration et de la communication reste de favoriser le partage de données entre les acteurs du One Health. Pour mieux définir, prévenir ou simuler le risque infectieux, il est indispensable d'être en mesure de croiser des données de surveillance humaines, animales et environnementales. En dépit de nombreuses initiatives de surveillance en santé humaine et animale, force est de constater qu'aujourd'hui les interconnections restent encore limitées du fait de méthodologies et d'indicateurs différents. La France dispose, par exemple, d'outils de surveillance performants en matière de lutte contre l'antibiorésistance, que ce soit chez l'homme (mission PRIMO, https://www.cpias-pdl.com/acco mpagnement/missions-nationales- $2 /$ mission SPARES, http://www.cpias-grand-est.fr/index.php/secteur-sanitaire/mi ssions-nationales/spares/, réseau ONERBA, http://onerba.org/), ou chez l'animal (réseau RESAPATH, https://resap ath.anses.fr/). Si ces réseaux de surveillance parviennent efficacement à produire des rapports permettant de faire un état des lieux de l'antibiorésistance, et de voir l'évolution de différents indicateurs, ils ne permettent pas à l'heure actuelle de caractériser, à l'appui des données de surveillance, les facteurs de risques 
permettant la transmission des résistances d'un réservoir à l'autre ni de prévoir ou simuler l'émergence de résistances. Leur approche reste cloisonnée. L'un des enjeux derrière la structuration de réseaux One Health est donc la création d'entrepôts de données de surveillance humaine, animale et environnementale permettant de faire des analyses de risque in silico. L'analyse de métadonnées One Health pourrait par exemple, via des outils de " machine learning ", permettre d'identifier des facteurs de risques environnementaux encore méconnus. L'interopérabilité de ces données hétérogènes doit reposer sur une démarche d'harmonisation des pratiques de surveillance entre secteurs et sur la définition d'indicateurs communs. Le croisement de ces données harmonisées pourrait ensuite se faire à la faveur de variables spatio-temporelles d'intérêt.

\section{Renforcer la surveillance environnementale}

L'environnement possède un rôle central dans la lutte One Health contre l'antibiorésistance. Il est non seulement un réservoir de gènes de résistance, un lieu privilégié pour les transferts horizontaux et concentre tous les polluants anthropiques. La lutte contre l'antibiorésistance ne se limite pas à la bonne utilisation des antibiotiques en santé humaine et animale. Elle doit s'accompagner de mesures pour réduire l'impact des antibiotiques sur l'environnement et préserver la qualité des eaux, sols et écosystèmes. En dépit du rôle central de l'environnement dans le spectre One Health, ce secteur est encore trop souvent négligé notamment en matière de surveillance. La surveillance environnementale peut pourtant être un puissant outil de suivi épidémiologique comme démontré récemment lors de la crise Covid-19. Le suivi du virus dans les eaux usées, via le réseau Obépine en France (Wurtzer et al. 2020), s'est révélé être un précieux indicateur pour évaluer la reprise épidémique localement et mener des actions de dépistages préventifs. Structurer et renforcer la surveillance environnementale est donc un enjeu crucial pour consolider les approches One Health existantes et mieux prévenir le risque épidémique. Néanmoins le renforcement de la surveillance environnementale se heurte à plusieurs barrières. Tout d'abord la fragmentation de ses acteurs. De nombreuses initiatives de surveillance environnementale existent mais souvent cantonnées à un écosystème particulier (eaux de rivières, eaux stagnantes, sols, ...). Un des enjeux est là encore de parvenir à rassembler tous ces acteurs autour d'enjeux communs et à structurer de véritables réseaux de surveillance environnementaux intégrant différents écosystèmes d'intérêt. De plus, il est nécessaire de réfléchir à des indicateurs pertinents à la fois pour relier la surveillance dans l'environnement à celle réalisée chez l'homme ou l'animal (par exemple Escherichia coli comme dans le protocole Tricycle de l'OMS), mais aussi pour surveiller spécifiquement et de façon longitudinale la résistance dans l'environnement. Pour ce dernier point, des indicateurs génomiques globaux pourraient être de meilleurs indicateurs que des gènes de résistance particuliers (Huijbers et al. 2019). Enfin, dans cette surveillance environnementale, il convient de ne pas négliger certains écosystèmes notamment les plantes, soumises à des résidus antibiotiques a les épandages et dont le rôle dans la transmission des résistances est souvent oublié (Destoumieux-Garzón et al. 2018).

\section{Renforcer les démarches éducatives et de forma- tion autour du One Health}

La systématisation du One Health en pratique passe avant tout par la formation. Il convient d'une part de renforcer les compétences des professionnels du One Health via des outils de formation (séminaire, MOOC...) ou des forums de rencontre et d'échange de pratiques. D'autre part, il est également important de créer des formations pour les jeunes professionnels issus des différentes filières spécifiques, santé humaine, santé animale et environnement, centrées autour des enjeux du One Health. Dans les deux cas, il s'agit d'identifier des compétences clés indispensables aux praticiens du One Health et à les transmettre aux acteurs impliqués. Dans ce cadre, il est impératif d'impliquer les sciences sociales pour identifier les barrières et faciliter l'assimilation de ces compétences clés et leur application pratique.

\section{Sensibiliser le grand public au One Health}

Pour lancer une dynamique One Health durable, il est indispensable de sensibiliser le grand public à ce concept. Dans un monde où la société est de plus en plus sensible aux enjeux climatiques et environnementaux, l'interdépendance entre humains, animaux et environnement se fait de plus en plus évidente. Il est notamment important de capitaliser sur la crise du Covid-19 pour sensibiliser au risque posé par les zoonoses et au One Health. Le grand public doit être en mesure de s'emparer de ces sujets pour créer la dynamique nécessaire à l'institutionnalisation du One Health à tous les niveaux. Cette sensibilisation passe par des actions de vulgarisation et la création de contenus interactifs accessibles à tous via les réseaux sociaux notamment. Elle repose également sur l'implication des mass médias pour véhiculer, au plus grand nombre, les enjeux du One Health. Ici encore, les sciences sociales et du langage ont un rôle à jouer pour clarifier et faciliter la transmission du message véhiculé.

\section{CONCLUSION : VERS UN ONE HEALTH PLUS SYSTÉMIQUE ET INSTITUTIONNALISÉ}

Au cours des 20 dernières années, le One Health a réussi à fédérer de nombreuses disciplines autour d'enjeux communs de santé publique. La profession vétérinaire a grandement contribué à cette dynamique vertueuse avec des accomplissements majeurs notamment dans la diminution des prescriptions d'antibiotiques. En dépit de ces progrès et de la multiplication des approches multisectorielles, le One Health ne parvient toujours pas à s'institutionnaliser. Bien que régulièrement discuté à la faveur d'épidémies, il peine encore à s'ancrer dans les pratiques.

Pour devenir plus systémique, le One Healthh doit avant tout apprendre à se structurer et à fédérer ses acteurs autour d'enjeux communs tels que l'échange de bonnes pratiques ou de 
données. Il doit également apprendre à mieux incorporer la dimension environnementale dans son action. Enfin, il doit capitaliser sur l'éducation et la sensibilisation pour créer une dynamique One Health durable capable de s'ancrer dans les pratiques et de mobiliser le grand public autour d'enjeux majeurs de santé publique.

La France a bien su se saisir de ses enjeux. En lien avec la feuille de route interministérielle de maîtrise de la résistance aux antibiotiques, les différents ministères et agences impliqués travaillent de concert pour dérouler les différentes actions du plan national One Health de lutte contre l'antibiorésistance.
Parmi les perspectives intéressantes, on peut notamment se réjouir de voir s'amorcer en France la construction (i) d'un méta-réseau professionnel One Health rassemblant les principaux acteurs des domaines humain, animal et environnemental, impliqués dans la lutte contre l'antibiorésistance (ii) d'une plateforme multi-omics One Health et (iii) d'un observatoire des discours et des normes liées à l'antibiorésistance. Ces outils structurants devraient permettre de renforcer les synergies et d'ancrer les pratiques One Health à l'échelle nationale tout en permettant à la France de renforcer sa position dans le domaine sur la scène européenne voire mondiale.

\section{BIBLIOGRAPHIE}

- ANSES. Résapath Réseau d'épidémiosurveillance de l'antibiorésistance des bactéries pathogènes animales Bilan 2019, 2020. Disponible à : https://r esapath.anses.fr/resapath_uploadfiles / files/Documents/2019 RESAPATH \%20Rapport\%20 A nnuel.pdf (consulté le 13 juillet 2021).

- ANSM. L'évolution des consommations d'antibiotiques en France entre 2000 et 2013, 2014. Disponible à : http:/ /dev4-afssaps-marche2017.int egra.fr/var/ansm_site/storage/orig i n a $1 /$ a p p lic a t i o n / 28 f 39 921e2a8d8368a1fbfcdc496bd88.pdf (consulté le 13 juillet 2021).

- Booth M. Climate change and the neglected tropical diseases. Adv Parasitol. 2018;100: 39-126.

- Cassini, A, Högberg LD, Plachouras D, Quattrocchi A, Hoxha A, Simonsen GS et al. Attributable deaths and disabilityadjusted life-years caused by infections with antibiotic-resistant bacteria in the EU and the European Economic Area in 2015: a population-level modelling analysis. Lancet Infect Dis. 2019; 19: 56-66.

- Chiesa F, Tomassone L, Savic S, Bellato A, Mihalca AD, Modry D et al. A Survey on One Health perception and experiences in Europe and neighboring areas. Front Public Health. 2021; 9: 609949.

- Comité interministériel pour la Santé. Feuille de route interministérielle visant à maîtriser l'antibiorésistance, 2016. Disponible à : https://solidarites-sant e.gouv.fr/IMG/pdf/feuille_de_route_a ntibioresistance_nov_2016.pdf (consulté le 10 juin 2021).
- Commission Européenne. Stratégie communautaire de lutte contre la résistance antimicrobienne, 2001. Disponible à : https://eur-lex.eur opa.eu/legal-content/FR/TXT/H $\mathrm{TML} /$ ? uri=LEGISSUM : c 1156 8\&from=FR (consulté le 10 juin 2021).

- Commission Européenne. Conclusions du Conseil sur les prochaines étapes de la lutte contre la résistance aux antimicrobiens dans le cadre du concept "Une seule santé", 2016. Disponible à : https://www.g7uk.org/g7-health-mini sters-meeting-communique-oxford4-june-2021/(consulté le 10 juin 2021).

- Commission Européenne. A European One Health Action Plan against Antimicrobial Resistance (AMR), 2017. Disponible à : https://ec.eur opa.eu/health/sites/default/files/ant imicrobial_resistance/docs/amr_2017_ action-plan.pdf (consulté le 10 juin 2021).

- Destoumieux-Garzón D, Mavingui P, Boetsch G, Boissier J, Darriet F, Duboz, $\mathrm{P}$ et al. The One Health concept: 10 years old and a long road ahead. Front Vet Sci. 2018; 5:14.

- G7. G7 Health Ministers' Communique, 2021. Disponible à : https://www.g7uk.org/g7-health-mini sters-meeting-communique-oxford4-june-2021/(consulté le 10 juin 2021). Garcia-Graells C, Antoine J, Larsen J, - Catry B, Skov R, Denis O. Livestock veterinarians at high risk of acquiring methicillin-resistant Staphylococcus aureus ST398. Epidemiol Infect. 2012;140: 383-9.

- Moulin G, Chevance A, Méheust D, Jouy E, Jarrige N, Lupo A et al. . Ecoanti- bio 2017: bilan, évolution des consommations et des résistances, pourquoi un Ecoantibio 2 ? Bull. GTV. 2017 ; 88 : 15-25.

- Haenni M, Saras E, Châtre P, Médaille C, Bes M, Madec JY et al. A USA300 variant and other human-related methicillin-resistant Staphylococcus aureus strains infecting cats and dogs in France. J Antimicrob Chemother. 2012; 67: 326-9.

- Huijbers PMC, Flach CF, Larsson DGJ. A conceptual framework for the environmental surveillance of antibiotics and antibiotic resistance. Environ Int. 2019; 130: 104880.

- Jeggo M, Mackenzie JS, Atlas Ronald M, Maloy S. Defining the Future of One Health. Microbiology Spectrum 2014;2, 2.1.12.

- Jones KE, Patel NG, Levy MA, Storeygard A, Balk D, Gittleman JL et al. Global trends in emerging infectious diseases. Nature. 2008; 451:990-3.

- Köck R, Loth B, Köksal M, Schulte-Wülwer J, Harlizius J, Friedrich AW. Persistence of nasal colonization with livestock-associated methicillin-resistant Staphylococcus aureus in pig farmers after holidays from pig exposure. Appl Environ Microbiol; 2012;78: 4046-7.

- Mader R, Bourély C, Amat JP, Broens EM, Busani L, Callens,B., et al. Defining the scope of the European Antimicrobial Resistance Surveillance network in Veterinary medicine (EARS-Vet): a bottom-up and One Health approach. bioRxiv 2021a.03. 09.434124. doi: https://doi.org/10. $1101 / 2021.03 .09 .434124$ 
- Mader R, Damborg P, Amat, JP, Bengtsson B, Bourély C, Broens, et al. Building the European Antimicrobial Resistance Surveillance network in veterinary medicine (EARS-Vet). Eurosurveillance 2021b; 26: ): 2001359.

- Ministère chargé de la Santé. Plan national d'alerte sur les antibiotiques 2011-2016, 2011. Disponible à : https:/ /solidarites-sante.gouv.fr/IMG/ pdf/plan_antibiotiques_2011-2016_.pdf (consulté le 10 juin 2021).

- Ministère des affaires étrangères et européennes. Position française sur le concept "One Health/Une seule santé», 2011. Disponible à : https://www.dipl omatie.gouv.fr/IMG/pdf/Rapport_One _Health.pdf (consulté le 10 juin 2021).

- O’Neill J. Tackling drug-resistant infections globally: final report and recommendations, 2016. Disponible à : https://amr-review.org/sites/default /files/160518_Final\%20paper_with\% 20cover.pdf (consulté le 13 juillet 2021).

- Organisation for Economic Co-operation and Development (OECD. Stemming the Superbug Tide, 2018. Disponible à : https://doi.org/10.1787/9789264 307599-en (consulté le 10 juin 2021).

- Poirel L, Berçot B, Millemann Y, Bonnin RA, Pannaux G, Nordmann P. Carbapenemase-producing Acinetobacter spp. in Cattle, France. Emerg Infect Dis. 2012; 18(3): 523-525.

- Salyer SJ, Silver R, Simone K, Barton
Behravesh C. Prioritizing zoonoses for global health capacity building-themes from One Health zoonotic disease workshops in 7 Countries, 2014-2016. Emerging Infectious Disease 2017; 23 : S55-S64.

- Santé Publique France. Antibiotiques et résistance bactérienne : une menace mondiale, des conséquences individuelles, 2019. Disponible à : https://www santepubliquefrance.fr/maladies-et-tra umatismes/infections-associees-auxsoins-et-resistance-aux-antibiotiques/resi stance-aux-antibiotiques/documents/ rapport-synthese/antibiotiques-et-resi stance-bacterienne-une-menace-mo ndiale-des-consequences-individuelles (consulté le 13 juillet 2021).

- White A, Hughes JM. Critical importance of a One Health approach to antimicrobial resistance. EcoHealth. 2019; 16:404-9.

- WHO. Global action plan on antimicrobial resistance, 2016. Disponible à : https://www.who.int/publications/i/ item/9789241509763 (consulté le 10 juin 2021).

- WHO. Liste OMS des antibiotiques d'importance critique pour la médecine humaine (liste CIA), 5e révision, 2019. Disponible à : https://apps.who.int/ iris/bitstream/handle/10665/325035/ WHO-NMH-FOS-FZD-19.1-fre.pdf?s equence $=9$ \& isAllowed $=y$ (consulté le 10 juin 2021).
- Wildlife Conservation Society (WCS). The Manhattan Principles, 2004. Disponible à : https://oneworldonehealth.wcs.org/About-Us/Mission/ The-Manhattan-Principles.aspx (consulté le 10 juin 2021).

- Woolhouse ME, Gowtage-Sequeria S. Host range and emerging and reemerging pathogens. Emerg Infect Dis; 2005; 11:1842-7.

- Wu Y, Peng Z, Yan Y, Hu J, Wang Y, Wang X et al. Current knowledge of COVID-19: advances, challenges and future perspectives. Biosaf Health. 2021; Jun 4.

- Wurtzer S, Waldman P, Ferrier-Rembert A, Frenois-Veyrat G, Mouchel JM, Boni $\mathrm{M}$ et al. Several forms of SARS-CoV-2 RNA can be detected in wastewaters: implication for wastewater-based epidemiology and risk assessment. Water Res. 2021; 198: 117183.

- Yaici L, Haenni M, Métayer V, Saras E, Mesbah Zekar F, Ayad M et al. Spread of ESBL/AmpC-producing Escherichia coli and Klebsiella pneumoniae in the community through ready-to-eat sandwiches in Algeria. Int J Food Microbiol. 2017; 245: 66-72.

- Zinsstag J, Crump L, Schelling E, Hattendorf J, Maidane YO, Ali KO et al. Climate change and One Health. FEMS Microbiol Lett. 2018;365: fny085. 